M. HEURS
T. MEIER $^{1}$
V.M. QUETSCHKE $^{1,2}$
B. WILLKE
I. FREITAG $^{3}$
K. DANZMANN

\section{Intensity and frequency noise reduction of a Nd:YAG NPRO via pump light stabilisation}

\author{
${ }^{1}$ Max-Planck-Institut für Gravitationsphysik (Albert-Einstein-Institut) \\ und Institut für Atom- und Molekülphysik, Abteilung Spektroskopie, \\ Universität Hannover, Callinstr. 38, 30167 Hannover, Germany \\ ${ }^{2}$ Currently University of Florida, Gainesville, USA \\ ${ }^{3}$ InnoLight GmbH, Garbsener Landstr. 10, 30419 Hannover, Germany
}

\begin{abstract}
Received: 2 March 2006/Revised version: 30 May 2006 (c) Springer-Verlag 2006

ABSTRACT We have shown that pump light intensity stabilisation of a single-mode laser diode pumped Nd:YAG non-planar ring oscillator (NPRO) results in significant intensity noise reduction of the NPRO, as well as frequency noise suppression in the same order of magnitude. This effect does not occur in conventional laser diode array pumped NPROs due to mode beating effects originating in the multi-mode pump. As opposed to individual intensity and frequency stabilisation, pump light stabilisation contributes a simplified stabilisation scheme for single-mode laser diode pumped NPROs for high precision applications.
\end{abstract}

PACS 42.55.Xi; 42.60.By; 42.60.Lh

\section{$1 \quad$ Introduction}

Due to their intrinsically high stability, laser diode pumped solid state lasers are perfectly suited as light sources for high precision metrology measurements, amongst them interferometric gravitational wave detectors [1-4]. The many requirements posed by these applications call for active stabilisation of the laser observables frequency, intensity and beam geometry. A well known problem is parasitic coupling [5], which often results in a worsening of one observable when another is stabilised.

The intensity noise of a Nd:YAG solid-state laser depends on various parameters, such as the properties of the laser medium, the noise and the spatial properties of the pump source, as well as vacuum fluctuations. In conventional laser diode array pumped NPROs the stabilisation of the pump intensity does not necessarily result in a reduction of NPRO intensity noise [6]. However, it has been shown [7] that frequency stabilisation of a single-mode laser diode pumped NPRO using the current lock technique [8] yields a high degree of frequency noise reduction with an equally high degree of simultaneous intensity noise suppression. This work deals with the intensity stabilisation of the pump light of this laser system, examining the possibilities posed by the coupling effect.

Fax: +49-511-762-2784, E-mail: michele.heurs@aei.mpg.de

\section{Experiments with a laser diode array}

Harb et al. [6] have shown that the intensity noise spectrum of an NPRO can be split into different regimes. For frequencies well below the resonant relaxation oscillations the intensity noise spectrum is dominated by pump intensity fluctuations. It would be intuitive to assume that a stabilisation of the pump intensity would result in a reduction of the NPRO intensity noise. Experiments were conducted on a laser diode array (LDA), the pump source for conventional NPROs, to investigate this assumption; the experiments and their results are described in the following subsections.

\subsection{Aperture effect in laser diode arrays}

Unavoidable spatial apertures occur when an LDA is used for end-pumping of an NPRO crystal. Due to the high divergence of the multi-mode LDA, the overlap between pump and laser mode in the NPRO cannot be perfect. This fact is exemplified in Fig. 1, which shows the beam profiles of the pump mode (of a fibre-coupled LDA) and the NPRO mode.

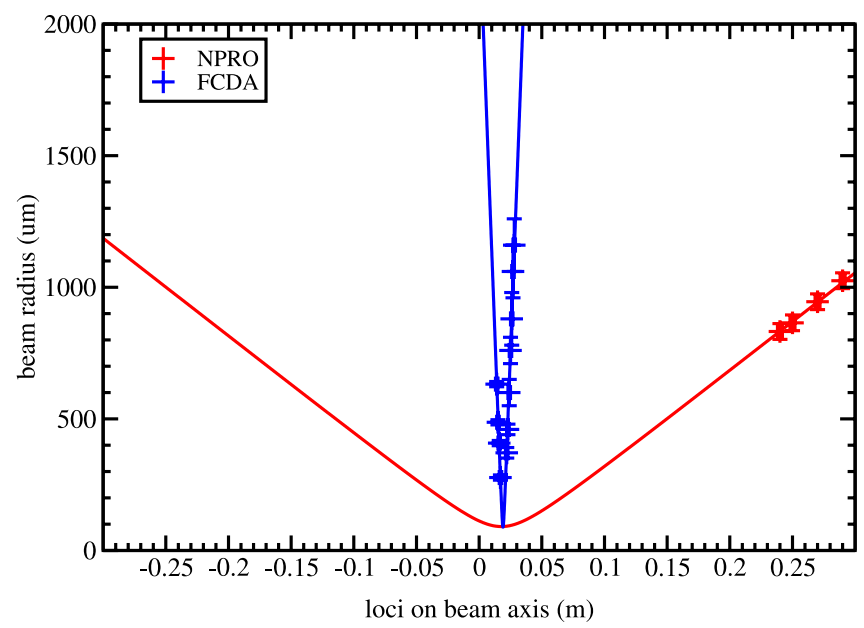

FIGURE 1 Measured beam profiles of the pump and the NPRO mode. The multi-mode beam emitted by the laser diode array and the TEM 00 NPRO laser mode are measured at various positions behind the front facette of the NPRO crystal (the crystal obviously has to be removed to measure the pump mode) 
As this work deals with the possibility of intensity stabilisation of an NPRO by means of intensity stabilisation of its pump source, it is crucial to determine the effects of an aperture in the LDA's beam path on its intensity noise. Therefore the intensity noise of an LDA was measured with different spatial apertures, but with constant drive current and fixed detected intensity. The experimental setup was realised in the following way:

An LDA (type 2Y81 from Osram Opto Semiconductors, [9]) was driven by a low-noise current source (InnoLight), resulting in an emitted light power of $1 \mathrm{~W}$. The light of the LDA was attenuated by an appropriate set of Schott neutral density filters. The light was then focussed onto a photodetector. A silicon photodiode with an active area of $41.3 \mathrm{~mm}^{2}$ (type IPL 10050, Thales Optronics) was used for detection of the intensity noise of the LDA. A transitional amplifier transformed the photocurrent into a proportional voltage. The $3 \mathrm{~dB}$ bandwidth of the photodetector was $\approx 1 \mathrm{MHz}$. An iris diaphragm was then introduced in the beam path and the filters were adjusted accordingly to ensure constant optical power on the photodetector. The same measurements were conducted with a different LDA (type 2F81), which is equipped with a fibre receptacle for an FC-PC fibre with a core diameter of $200 \mu \mathrm{m}$ and an SMA coupler [10]. The nominal output power of this LDA was $1.5 \mathrm{~W}$. The measurements of relative intensity noise were done with and without a fibre attached, respectively, again taking into account the different dc power levels on the photodetector by appropriately choosing the neutral density filters. For these measurements of relative intensity noise a spectrum analyser from Agilent Technologies (type 4395A) was used.

The results of these measurements are shown in Fig. 2. It is clearly visible that any kind of spatial aperture (diaphragm or fibre) introduced in the beam path of an LDA causes an increase in its relative intensity noise. This aperture effect is caused by the fact that the emission of the individual emitters of the LDA is anti-correlated. The emitters are electrically contacted in parallel, causing the current to vary over the in-

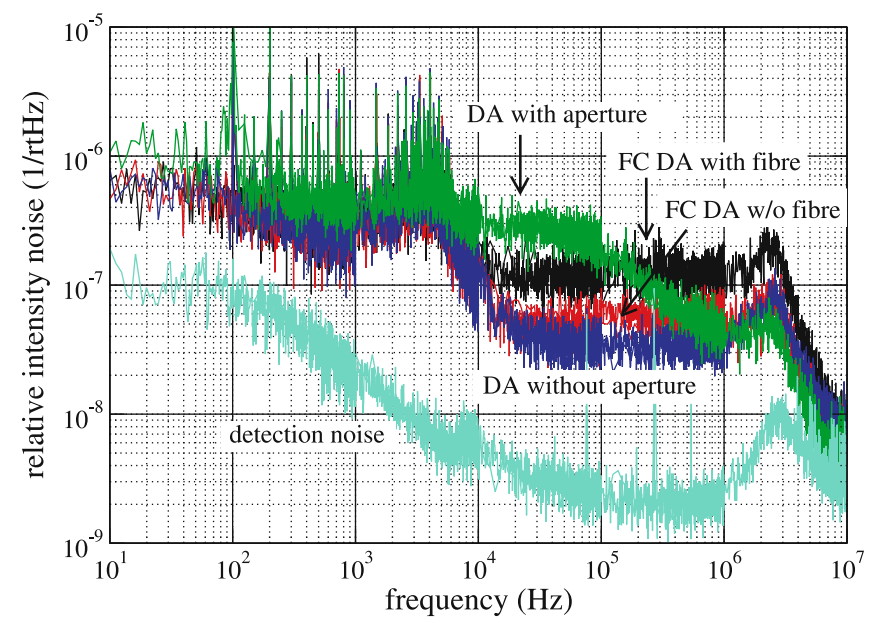

FIGURE 2 Relative intensity noise of a free running LDA with and without an aperture in the beam path. Introduction of any kind of aperture leads to higher intensity noise of the LDA. The large roll-off at frequencies above $\approx 1 \mathrm{MHz}$ is caused by the electronic response of the photodetector. DA: diode array; FC: fibre-coupled dividual emitters, which results in power fluctuations over the beam profile. The overall intensity is then as constant as the drive current, but the intensity varies across the beam profile. Detection of the complete profile then gives a lower intensity noise level than detection of only a fraction of the beam diameter. This result entails an important fact: even with constant drive current, unavoidable apertures in the pump beam cause excess pump intensity noise, which according to [6] gives rise to excess NPRO intensity noise. This fact and the possibilities of intensity stabilisation of the pump source are examined in the following section.

Due to its design the emission of the LDA is not diffraction limited. The spatial profile does not resemble a TEM ${ }_{00}$ Gaussian mode; images of the spatial profile of the free-beam LDA in near- and far-field are depicted in [6]. For our investigations concerning the pump light stabilisation of an LDA pumped NPRO we used a fibre-coupled LDA to obtain a roughly round pump mode. The emission of the fibre-coupled LDA exhibits a spatial profile as shown in Fig. 3. The stationary picture cannot reveal the observed strong intensity fluctuations of the profile. The large spatial and temporal variations of the intensity are most likely due to multi-path interference in the multi-mode fibre.

\subsection{Pump light stabilisation of a laser diode array pumped NPRO}

An electronic control loop (for a more detailed description see Sect. 3) was designed to obtain intensity stabilisation of a fibre-coupled LDA (type 2F81). Feedback to the pump current of the LDA was realised with a transistor current sink [11] which is contacted in parallel to the LDA. The light emitted by the fibre-coupled LDA was split up by a power beam splitter and detected by two identical photodetectors of the same design as described in Sect. 2.1. One beam was used as the signal for the pump light stabilisation electronics (the signal is therefore a measure of the in-loop intensity noise); the processed signal was fed back to the LDA via the current bypass end stage of the control electronics. The other detected beam was an independent measure of the out-of-loop intensity noise (with and without active stabilisation). The resulting in-loop and out-of-loop intensity noise of the LDA (with and without active pump light stabilisation, respectively) is shown in Fig. 4. The control bandwidth exceeds $100 \mathrm{kHz}$. A large in-loop intensity noise reduction of up to $40 \mathrm{~dB}$ is achieved; the out-of-loop reduction, though, is much weaker with only
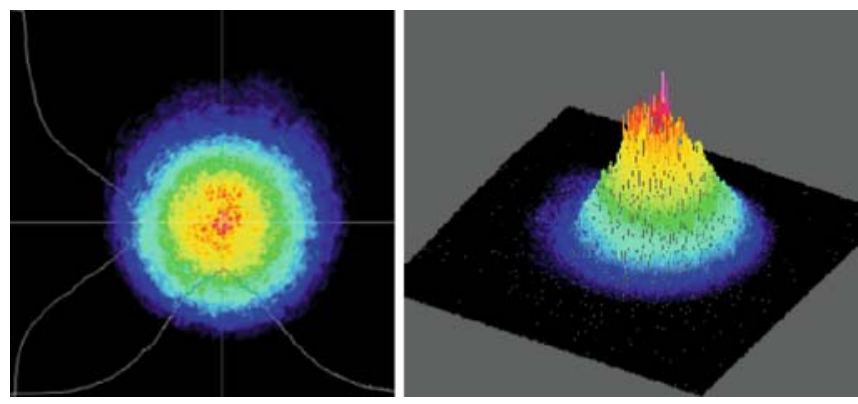

FIGURE 3 Image of the spatial distribution of the light emitted by a fibrecoupled LDA (type 2F81) (left: view from top, right: as seen under an angle). The distribution shows large temporal variations 


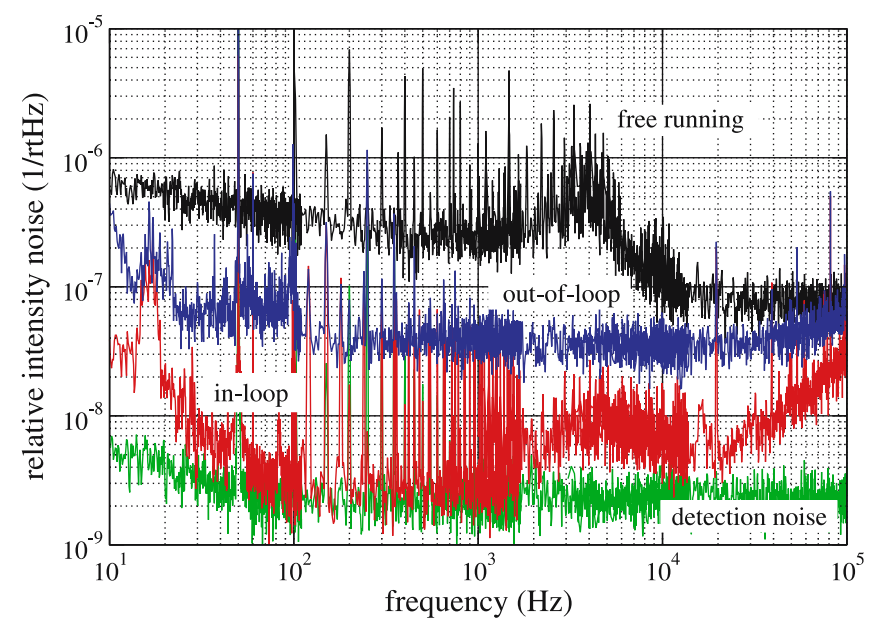

FIGURE 4 In-loop and out-of-loop relative intensity noise of a fibrecoupled LDA with and without active intensity stabilisation

$20 \mathrm{~dB}$ maximum. The reason for this discrepancy lies partly in the fact that due to inevitable apertures in the respective beam paths not all of the beam diameter is detected, resulting in the problems discussed above. Moreover the employed photodiodes exhibit an inhomogeneous responsivity, different for each diode. The inhomogeneities can exceed $2 \%$ across the active surface [12]. This gives rise to intensity noise when the beam profile changes over the surface of the detectors.

The same fibre-coupled LDA (type 2F81) was used to pump an NPRO crystal with reduced threshold. The emitted pump power was $1 \mathrm{~W}$ at $808 \mathrm{~nm}$. A fraction of the pump light (pick off beam from an uncoated glass wedge under nearnormal incidence) was detected with a silicon photodetector (as described in Sect. 2.1) and the remaining pump power $(\approx 900 \mathrm{~mW})$ was modematched into the NPRO crystal via an optical telescope. The detected pump light was used for pump intensity stabilisation. The complete experimental setup can be seen in Fig. 5.

Figure 6 shows the results of the pump intensity stabilisation on the NPRO intensity noise of a fibre-coupled LDA pumped NPRO.

Evidently, pump intensity stabilisation of a fibre-coupled LDA pumped NPRO does not result in a noticeable reduction of NPRO intensity noise, even though the active stabilisation obviously reduces the pump intensity noise, as can be seen in Fig. 4. The reason for this lies in the only partial overlap of pump and laser mode in the NPRO crystal. Spectral fluctua-

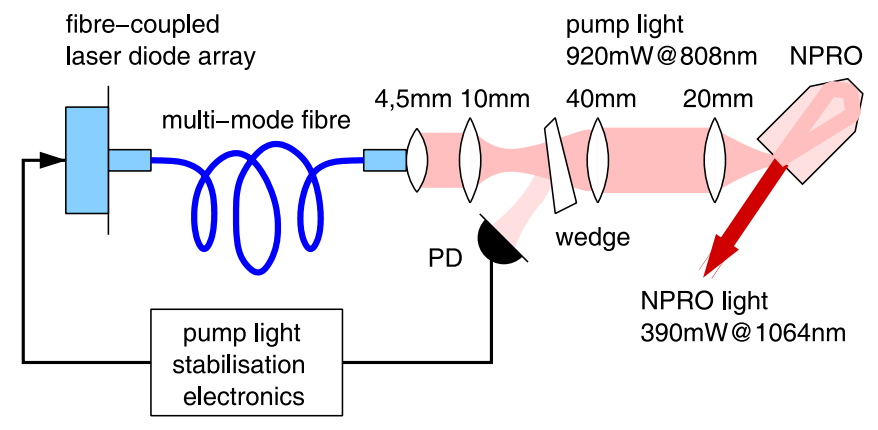

FIGURE 5 Experimental setup of the fibre-coupled LDA pumped NPRO

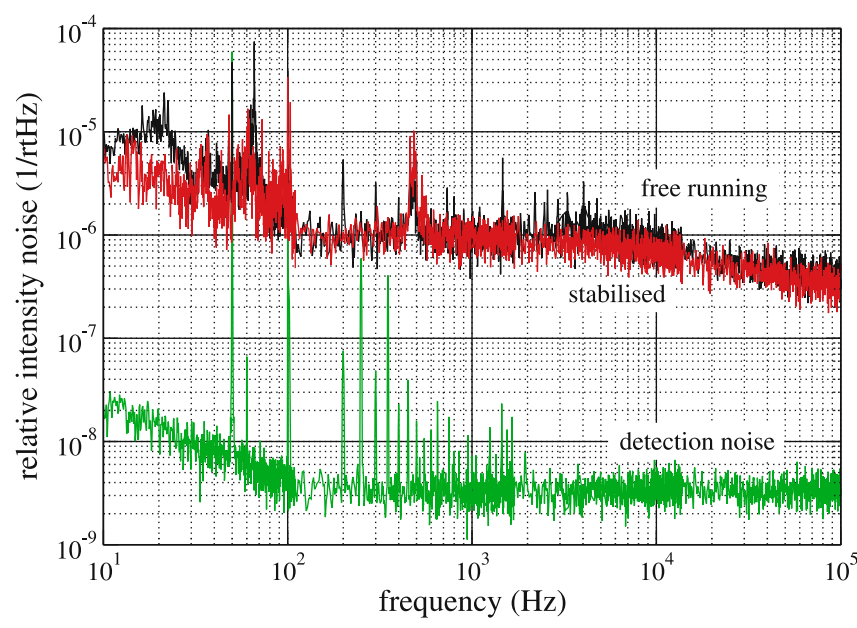

FIGURE 6 Free-running (black) and stabilised (red) relative intensity noise of a fibre-coupled LDA pumped NPRO. Pump light stabilisation obviously does not result in a reduction of intensity noise in this setup. The lower curve (green) represents detection noise

tions of the LD could in principle cause similar effects. From our current lock experiments $[7,8]$ we have evidence that this effect is not dominant, though (see discussion at the end of this section).

The spatial overlap of pump and laser mode in the LDA pumped NPRO can not be perfect due to the fact that the pump beam is not diffraction limited, as opposed to the NPRO beam. The pump light emitted by the LDA is focussed into the Nd:YAG crystal with a waist smaller than the NPRO waist to achieve mode selective pumping [13]. This leads to two distinct regions in the crystal: the overlap region, where pump intensity noise couples into NPRO intensity noise [6] via inversion as well as into NPRO frequency noise [14] via temperature induced changes of the optical length, and the non-overlap region, where deposition of pump power merely leads to a temperature change (coupling to NPRO frequency), but where the inversion is not depleted (thus having no effect on the NPRO intensity). The following simplified mathematical approach can help create an intuitive understanding of the complexity of the pump light intensity distribution. We omit the inherent time dependence of all variables for reasons of simplicity. The output power of an NPRO depends on the pump intensity $I_{\text {pump }}$, which for a laser diode array can be written as

$$
I_{\text {pump }}^{\mathrm{LDA}}\left(\boldsymbol{r}, v, j_{\mathrm{p}}\right)=I_{0}\left(j_{\mathrm{p}}\right)\left|\sum_{i=1}^{N} B_{i}\left(j_{\mathrm{p}}\right) f_{i}(\boldsymbol{r}, v)\right|^{2}
$$

with the normalisation condition

$$
\int \mathrm{d} \boldsymbol{r} \int \mathrm{d} v\left|\sum_{i=1}^{N} B_{i}\left(j_{\mathrm{p}}\right) f_{i}(\boldsymbol{r}, v)\right|^{2}=1
$$

where $\boldsymbol{r}$ is the coordinate in the observational plane, $v$ is the frequency of the pump light and $j_{\mathrm{p}}$ is the pump current. As stated in Sect. 2.1 the emission of the LDA is non-Gaussian. The functions $f_{i}$ describe the constituents of the spatial structure of the pump intensity; this structure can be arbitrarily 
complicated. The individual contributions to the spatial structure are weighted by the functions $B_{i}\left(j_{\mathrm{p}}\right)$ and should at least be a function of the pump current $j_{\mathrm{p}}$, but will most likely depend on other observables, too. $N$ denotes the total number of occurring modes $f_{i} . I_{0}\left(j_{\mathrm{p}}\right)$ denotes the overall intensity of the pump intensity distribution.

A change of the pump current $\Delta j_{\mathrm{p}}$ changes the overall intensity $I_{0}\left(j_{\mathrm{p}}\right)$, but also leads to a change of the individual mode contributions $B_{i}\left(j_{\mathrm{p}}\right)$. Therefore the effect of drive current variation $\Delta j_{\mathrm{p}}$ on the NPRO power is twofold, as the latter not only depends on the overall pump power, but strongly on the pump intensity distribution $I_{\text {pump }}^{\mathrm{LDA}}\left(\boldsymbol{r}, v, j_{\mathrm{p}}\right)$, too.

In contrast, a single-mode laser diode exhibits a Gaussian intensity distribution. Its overall power again varies with pump current, but its spatial distribution does not fluctuate. The implementation of a single-mode laser diode as NPRO pump source therefore leads to a simple linear relation between pump current and NPRO power.

The above is consistent with the fact that the transfer function from drive current of the multi-mode laser diode array to the NPRO power can not always be measured unambiguously and reproducibly, whereas that of the drive current of a singlemode pump to NPRO power is non-ambiguous. These considerations lead to the experiments described in Sect. 3 which employ a fibre-coupled single-mode pump source.

As stated above our current lock experiments $[7,8]$ give evidence that the effect of spectral overlap fluctuations between laser diode emission and Nd:YAG absorption is smaller than the above described spatial effect. The fluctuating LDA emission spectrum is absorbed by the wavelength dependent absorption of the Nd:YAG material. This leads to a varying amount of heat and inversion deposited in the spatial overlap region of pump and laser mode in the NPRO. The fluctuating inversion, however, is the cause of the NPRO intensity fluctuations. These fluctuations are correlated with the variation of the heat deposited in the laser material, which is the underlying detected observable in the current lock technique. In current lock the stabilised quantity is in fact the deposited heat, which in turn leads to a stabilisation of the laser frequency. As the amplitude noise reduction of an LDA pumped NPRO with active current lock is negligible [8] we can conclude that the varying spectral overlap is not the dominant effect for the experiment discussed here.

\section{Experiments with a single-mode laser diode pumped NPRO}

Based on the deductions in the above section we constructed a model laser system to further investigate the possibilities of NPRO intensity stabilisation via pump light intensity stabilisation. It consists of an NPRO crystal with reduced threshold pumped by a fibre-coupled single-mode laser diode (SMLD) pump source. The system is labelled singlemode laser diode pumped NPRO (SM-NPRO).

The SM-NPRO (InnoLight) uses a single-mode laser diode pump module constructed on a microbank-system by Schäfter \& Kirchhoff as a pump source. The pump module consists of a single-mode laser diode from SDL/JDS Uniphase, type 5422-H1-810 [15]. Due to technical reasons we pumped the NPRO at $813 \mathrm{~nm}$ (instead of the dominant absorption maximum at $808 \mathrm{~nm}$ ).
Single mode operation of the pump laser diode itself is only guaranteed if the pump current does not exceed a certain limit; the power of the emitted light is $150 \mathrm{~mW}$ at a pump current of $167 \mathrm{~mA}$, the slope efficiency of the laser diode without fibre coupling is $1.02 \mathrm{~W} / \mathrm{A}$ [15].

The pump light power of $70 \mathrm{~mW}$ emitted by the pump module behind the fibre coupling is passed through an optical telescope which matches the beam to the NPRO crystal Eigenmode. The mode matching was verified with a CCD beam analyser system, and the pump light alignment was optimised for output power. When the NPRO is pumped by the singlemode pump source with a pump power of $70 \mathrm{~mW}$ at $813 \mathrm{~nm}$, the laser emits $\approx 10 \mathrm{~mW}$ of laser light at $1064 \mathrm{~nm}$. This corresponds to an optical-optical slope efficiency of $\approx 50 \%$.

The internal monitor photodiode of the single-mode pump laser diode [15] is used to detect a small ratio of the pump light $(P \approx 100 \mu \mathrm{W})$. For small changes this photocurrent is proportional to the pump light intensity and is converted into a voltage by a transitional amplifier. The generated signal is then passed through an electronic control loop (pump light stabilisation electronics) which is composed of a PI-servo to frequency-dependently amplify the signal and a current bypass end stage to change the power of the pump laser diode. A "modulation box" (consisting of decoupling coils and isolated connectors) constructed directly next to the connectors of the laser diode enables direct pump current modulation whilst ensuring short wire lengths and thereby evading possibly fatal transients on the supply current and stray pick-up. With this scheme we were able to achieve control bandwidths of up to $70 \mathrm{kHz}$ (see Fig. 7). A passive current sink as opposed to an active current driver provides higher safety against accidental reverse biasing of the laser diode, which would be detrimental, as well as transients.

The signal of the internal monitor photodiode corresponds to the in-loop intensity noise of the pump laser diode and is shown in Fig. 7. The resulting intensity noise of the NPRO light is measured by direct detection of a pick off of the NPRO beam (again obtained with an uncoated glass wedge under near-normal incidence). The fast photodetector used for this

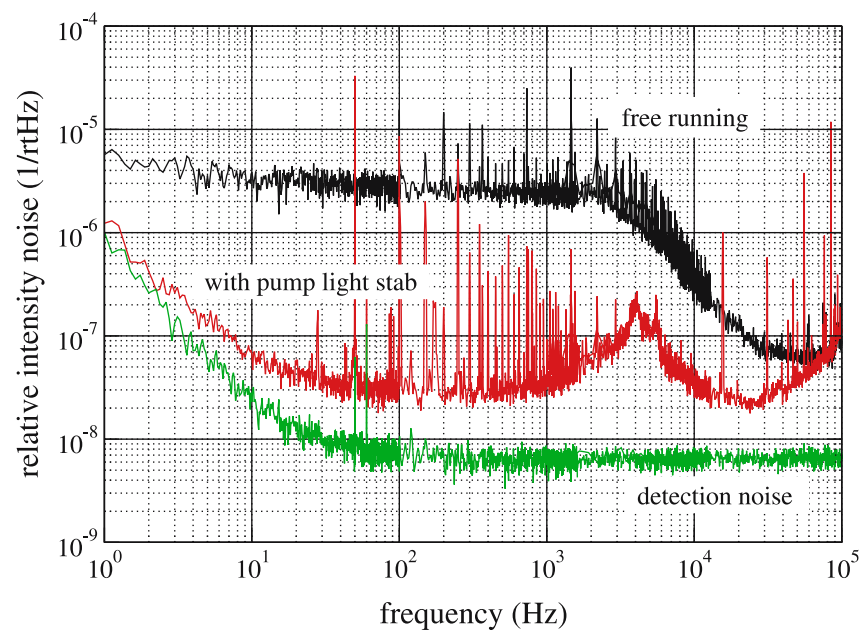

FIGURE 7 Intensity noise of the single-mode laser diode with and without active intensity stabilisation. The broad peak visible at $5 \mathrm{kHz}$ in the stabilised intensity stems from a resonance in the current driver 
measurement employs a $2 \mathrm{~mm}$ diameter InGaAs-PIN photodiode from EG\&G (type C 30642) in a photodetector circuit with high electronic bandwidth ( $>20 \mathrm{MHz})$. It can be seen in Fig. 7 that the intensity stabilisation of the single-mode laser diode results in an in-loop intensity noise reduction of up to $40 \mathrm{~dB}$ with a control bandwidth of around $70 \mathrm{kHz}$. The measurements shown in Figs. 7-9 were conducted with a low input noise spectrum analyser from Stanford Research (type SRT785). Structures visible in the spectrum are mainly caused by power line noise.

We detected $<500 \mu \mathrm{W}$ of the SM-NPRO light power with the above described InGaAs-photodetector; the measured free-running relative intensity noise (RIN), shown in Fig. 8, corresponds to a factor of up to 1000 above shot noise, which lies at $2.8 \times 10^{-8} / \sqrt{\mathrm{Hz}}$. Figure 8 clearly shows that the intensity stabilisation of the single-mode pump source of the SM-NPRO results in a significant reduction of SM-NPRO intensity noise by up to $26 \mathrm{~dB}$ at $2 \mathrm{kHz}$. The resonance structures in the spectrum stem from power line noise as well as acoustic noise coupling into the detection in the frequency band from $100 \mathrm{~Hz}$ to $1 \mathrm{kHz}$. The increase in intensity noise at high frequencies is caused by the resonant relaxation oscillation at $\approx 120 \mathrm{kHz}$. This also limits the bandwidth of the intensity noise reduction, as other noise processes become dominant there [6].

In [7] we showed that frequency stabilisation of the SMNPRO via feedback to the pump current of the pump source resulted in simultaneous frequency and intensity noise reduction of the SM-NPRO. Given the fact that pump intensity stabilisation in the SM-NPRO yields a large degree of intensity noise reduction of the SM-NPRO in this work (as opposed to the experiments with the LDA, see Sect. 2.2), it is of high interest to determine whether there is a further coupling effect to the SM-NPRO frequency noise. For this experiment the light of the SM-NPRO is phase-modulated by a New Focus electrooptical modulator (type 4003-M) resonant at 29.02 MHz. The phase-modulated light is modematched via a telescope into an optical cavity. This cavity is a three-mirror ring resonator built on an aluminum spacer with a piezoelectric crystal contacted

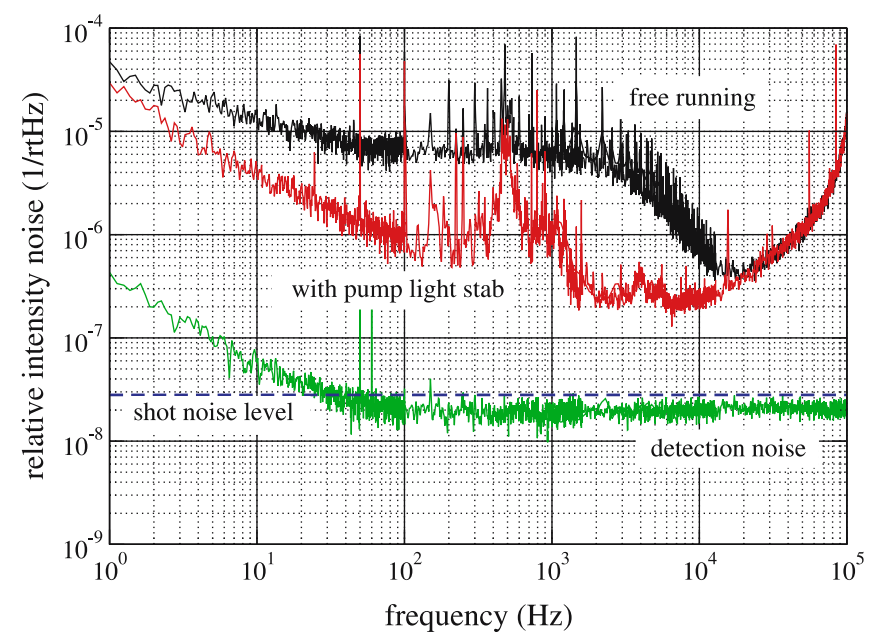

FIGURE 8 Intensity noise of the SM-NPRO with and without active intensity stabilisation. The shot noise level of the detected NPRO light lies at $2.8 \times 10^{-8} / \sqrt{\mathrm{Hz}}$ and is indicated by the dashed line to one mirror [16]. With the piezoelectric actuator the length, i.e. the resonance frequency of the ring cavity can be tuned. Stabilisation of the length of the optical resonator to the frequency of the SM-NPRO is achieved via the Pound-DreverHall method [17] and feedback to the piezoelectric transducer of the resonator. With this setup the frequency noise of the SM-NPRO (free-running as well as with active pump light stabilisation) is measured. As the tunable ring cavity is very susceptible to air currents and vibrations a vacuum chamber was constructed in which the ring cavity is placed in order to eliminate these disturbances and to facilitate the frequency noise measurements. When the cavity is stabilised to the laser, the feedback signal to the actuator of the cavity is a measure of the frequency noise of the laser for frequencies below unity gain of the Pound-Drever-Hall lock. The measured frequency noise with and without active pump light stabilisation is shown in Fig. 9. A reduction of the free-running frequency noise by approx. $20 \mathrm{~dB}$ is clearly visible, which corresponds to the reduction of the SM-NPRO intensity noise with pump light stabilisation. Noise coupling into the non-common beam paths (between intensity noise detection and frequency noise measurement) is the reason for the lesser frequency noise reduction as well as the reduced bandwidth, compared to the degree of intensity noise reduction. Acoustic noise coupling into the detection path leads to the structures visible in the spectrum at frequencies between $100 \mathrm{~Hz}$ and $1 \mathrm{kHz}$.

\section{$4 \quad$ Conclusion}

The intensity noise of a laser diode pumped NPRO is reduced by intensity stabilisation of its single-mode pump source via feedback to the pump current (pump light stabilisation). The control of the spatially well defined single-mode laser diode simultaneously leads to a significant reduction of the NPRO frequency noise. The pump intensity noise is a common cause for both the intensity and the frequency noise of the NPRO: below the resonant relaxation oscillations pump light fluctuations are the dominating cause for NPRO intensity noise [6] as well as for NPRO frequency noise [14] driven

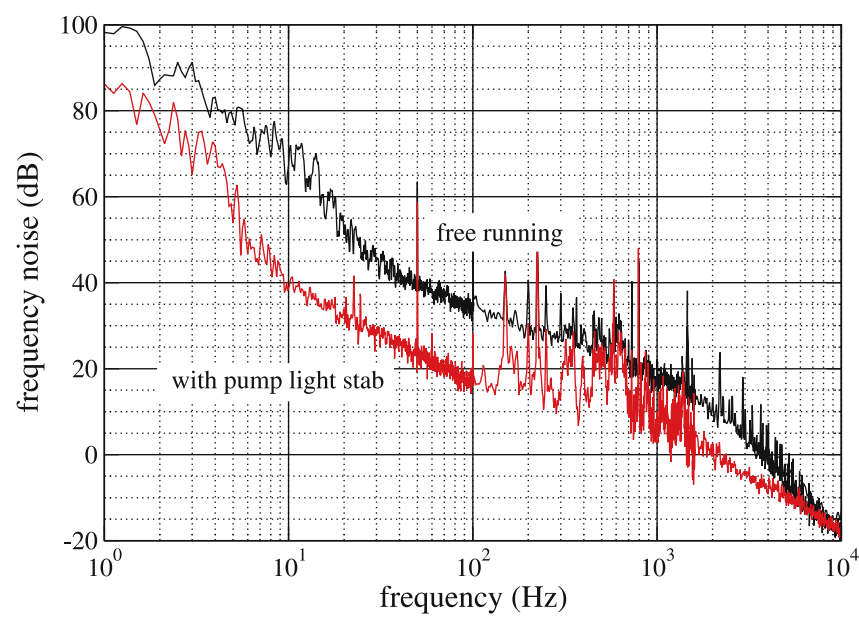

FIGURE 9 Frequency noise of the SM-NPRO with and without active intensity stabilisation. A simultaneous reduction of frequency noise with active pump light stabilisation on the order of $20 \mathrm{~dB}$ is clearly visible in a wide frequency band 
by the deposited heat in the laser active material, which consequently leads to fluctuations of the optical path length. For a multi-mode laser diode array pump source these fluctuations lead to an unpredictable effect on both NPRO intensity and frequency, depending on the locus of the absorption and the frequency distribution of the pump light. In contrast, for a different pumping scheme a single-mode pump source with one dominating frequency and a well-defined spatial profile leads to a defined effect on the NPRO intensity and frequency at every position in the NPRO crystal, and consequently allows for simultaneous stabilisation of both observables. The stabilisation technique developed in this work establishes a new possibility for simultaneous intensity and frequency stabilisation of Nd:YAG NPROs by use of only one actuator, the pump current. Rate equation simulations in our group yield quantitative results which are consistent with [6].

The pump intensity stabilisation experiments on a system with a different pump geometry, the laser diode array pumped NPRO, did not result in intensity noise reduction of the NPRO. However, a commercially available control electronics which detects a fraction of the NPRO intensity and feeds back to the pump current (a so-called "noise eater") can be used to suppress the NPROs resonant relaxation oscillations. A small, simultaneously occurring reduction of frequency noise has been shown in laser diode array pumped NPROs [18]. Following up on this, noise eater experiments have been conducted in our group showing that significant simultaneous suppression of frequency noise in a single-mode laser diode pumped NPRO can be achieved. These experiments are the subject of a future publication.

\section{REFERENCES}

1 B. Willke and the GEO 600-Team, Class. Quantum Grav. 21, 417 (2004)

2 B. Abbott and the LIGO Science Collaboration, Nucl. Instrum. Methods Phys. Res. A 517, 154 (2004)

3 F. Acernese and the VIRGO collaboration, Class. Quantum Grav. 21, 385 (2004)

4 M. Ando and the TAMA collaboration, Class. Quantum Grav. 19, 1409 (2002)

5 V.M. Quetschke, PhD thesis (Hannover, 2003)

6 C.C. Harb, T.C. Ralph, E.H. Huntington, D.E. McClelland, H.-A. Bachor, I. Freitag, J. Opt. Soc. Am. B 14, 2936 (1997)

7 M. Heurs, V.M. Quetschke, B. Willke, K. Danzmann, I. Freitag, Opt. Lett. 29, 2148 (2004)

8 B. Willke, O.S. Brozek, K. Danzmann, V. Quetschke, S. Gossler, Opt. Lett. 25, 1019 (2000)

9 Osram Opto Semiconductors, Datasheet, SPL 2Yxx

10 Osram Opto Semiconductors, Datasheet, SPL 2Fxx

11 R.S. Abbott, P.J. King, Rev. Sci. Instrum. 72, 1346 (2001)

12 F. Seifert, diploma thesis (Hannover, 2002)

13 Ingo Freitag, PhD thesis (Hannover, 1994)

14 T. Day, PhD thesis (Stanford, 1990)

15 JDS Uniphase, Datasheet, 5422-H1-810

16 B. Willke, N. Uehara, E.K. Gustafson, R.L. Byer, P.J. King, S.U. Seel, R.L. Savage, Opt. Lett. 23, 1704 (1998)

17 R.W.P. Drever, J.L. Hall, F.V. Kowalski, J. Hough, G.M. Ford, A.J. Munley, H. Ward, Appl. Phys. B 31, 97 (1983)

18 O.S. Brozek, PhD thesis (Hannover, 1999) 